ITM Web of Conferences 2, 06001 (2014)

DOI: $10.1051 /$ itmconf/20140206001

(C) Owned by the authors, published by EDP Sciences, 2014

\title{
Actuator disk modeling of the Mexico rotor with OpenFOAM*
}

\author{
A. Jeromin ${ }^{3}$, A. Bentamy ${ }^{2, a}$, and A.P. Schaffarczyk ${ }^{1, b}$ \\ ${ }^{1}$ CEwind eG and University of Applied Sciences Kiel, Grenzstr. 3, 24149 Kiel, Germany \\ ${ }^{2}$ School of Engineering and Science, Al Akhawayn University, 53000 Ifrane, Morocco \\ ${ }^{3}$ Forschungs- und Entwicklungszentrum FH Kiel GmbH, Schwentinestr., 24149 Kiel, Germany
}

\begin{abstract}
The implementation of an actuator disk with prescribed constant load for OpenFOAM was first presented by Svenning. In our presentation it was enhanced to compute local loads from local velocities by given aerodynamic lift and drag coefficients. The new model was then verified using the so called MEXICO rotor. Extensive comparisons to the experiments and other simulations were performed. The results for the thrust force was comparable to BEm and measurement wereas torque for the separated case $(25 \mathrm{~m} / \mathrm{s}$ inflow velocity) gave rather wide-spreading results.
\end{abstract}

\section{Introduction}

The application of computational methods in the design of wind turbines is common practice. It started in the mids of the 19th century by applying integral momentum theory and were then gradually enhanced by further analytical models like the blade element method (BEM). In this model the blade is devided into radial sections and all loads were averaged in circumferential direction. Modeling a rotor of a wind turbine by now is performed typically in the framwork of computational fluid dynamics (CFD) were only the geometry of the problem and the underlying equations of fluid mechnaics are to be used, which are assuemd to be the Navier-Stokes-Equations. This is espeacially difficult when the flow becomes turbulent. A myriad of engineering models have been developed with various stages of accuracy and computational effort. For a more detailled full account of Wind Turbine Aerodynamics see [1].

In contrast to the experiments BEM does not provide any information on the velocities in the nearby flow field. As a somewhat intermediate approach between BEM and full-sclae 3D RANS modeling, the actuator disk model (AD) is particulary appropriate. The surrounding flow field is modeled with CFD and the rotor is represented in a small volume where the forces from the blades have to be converted to appropriate volume forces.

The first attempt for implementing an AD model in OpenFOAM was conductes by Svenning [2] in his MSC-thesis. Here, the forces for thrust and torque were used to compute the volume source in each computational cell. This attempt works well when a constant loaded rotor was assumed. For a rotor in

\footnotetext{
*First Symposium on OpenFOAM in Wind Energy, Oldenburg, Germany, 20/21 march 2013, Corrected version 23 july 2013

ae-mail: a . bentamy@aui.ma

be-mail: alois.schaffarczyk@fh-kiel.de
}

This is an Open Access article distributed under the terms of the Creative Commons Attribution License 2.0, which permits unrestricted use, distribution, and reproduction in any medium, provided the original work is properly cited. 
a wind farm thrust and torque are dependent on the velocity distribution over the full disk area. Due to overlapping effects of wind turbines upstream the velocities might not be constant over the rotor disk.

To be able to include Wind-tunnel effects, a model was developed by Mahmoodi [3] using the commercial CFD-package FLUENT. Here the open flow as well as the wind-tunnel constained one could be simulated.

In the model presented we combine the framework of Svenning with the representation of Mahmoodi. A small but important improvement was, that the geometric and aerodynamic description could be read in as tables from files, so the error-prone step to parametrize the data could be skipped.

To test the new model a well documented and investigated experiment was used for comparison. The so-called MEXICO rotor was tested at different conditions in the German-Dutch wind tunnel (DNWLLF) in 2006, pressures on the blades and velocities in the field are available.

In the following sections first the MEXICO experiment is described insome detail, then the reference models will be described (Sect. 2). Our OpenFOAM implementation is described in detail in Section 3. Results are shown in Section 4, and the paper finishes with conclusions in Section 5.

\section{Description of the reference models}

In the MEXICO project [4] experiments were performed in the largest German-Dutch wind tunnel using a 3-bladed rotor of $4.5 \mathrm{~m}$. After the experiments were finished and much data was gathered, the IEA Task 29 "MexNext" was started to compare experiments, blade element models, actuator disk models, lifting line models, computational fluid dynamics (CFD) and other methods in aerodynamics for wind turbines. In this task a common base was defined for the comparisons by specifying boundary conditions like wind speed, tip speed ratio etc..

Three of these sets will also be the basis for the comparison in this study. In all three sets the rotational speed of the wind turbine will be 424 RPM. The inflow wind speeds are set to $10 \mathrm{~m} / \mathrm{s}, 15 \mathrm{~m} / \mathrm{s}$ and $24 \mathrm{~m} / \mathrm{s}$ or to tip speed ratios (tsr) 10, 6.7 (design tsr) and 4.2, respectively. The pitch angle was $-2.3^{\circ}$ in all three cases.

In the following sections the setup of the experiment and of each simulation model will be explained in detail.

\subsection{Experiments}

The experiments were performed with a three-bladed wind turbine rotor of $4.5 \mathrm{~m}$ diameter in the German-Dutch wind tunnel (DNW). The rotor blades were assembled from three different airfoils (see fig. 1). Between these airfoils transfer zones allow for a smooth transition.

Thrust and toque were measured by a balance in the tower foot. The blade loads were integrated from pressure measurements at five radial positions within the defined airfoil sections. To measure the velocity profiles the particle image velocimetry (PIV) was used.

For the radial velocity profiles different azimuthal positions of the blades were considered. For azimuth $\Psi=0^{\circ}$ blade 1 was pointing up (12 o'clock position) while the PIV-sheet was at 9 o'clock position (see fig 2). In total six azimuthal positions were taken into account at $\Psi=\left(0^{\circ}, 20^{\circ}, 40^{\circ}, 60^{\circ}\right.$, $80^{\circ}, 100^{\circ}, 120^{\circ}$ ). The last azimuthal position was exactly when blade 2 was at 12 o'clock and used to cross-check the quality of the measurements. In this study, only the $\Psi=0^{\circ}$ azimuthal position was considered.

Axial velocity profiles were extracted from the center of the PIV-sheets at radius $r=1.37 \mathrm{~m}$ and $r=1.84 \mathrm{~m}(r / R=61 \%$ and $82 \%)$. The available data range extended from $x=-4.33 \mathrm{~m}(=$ $1.92 R$ upstream) to $x=5.73 \mathrm{~m}(=2.54 R$ downstream $)$. The azimuth position of the axial traverse was at $\Psi=0^{\circ}$. 


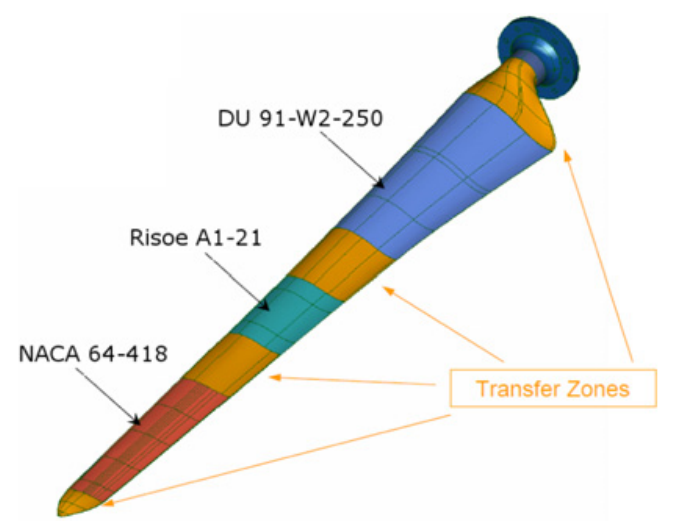

Figure 1. The MEXICO blade consists of three different airfoils. The shapes of the airfoils were merged in the transfer zones. (OECN).

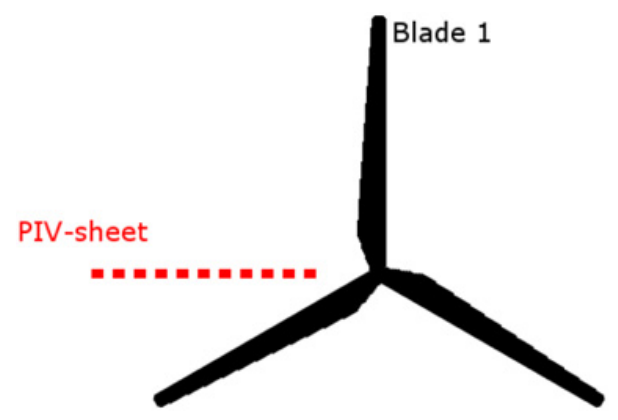

Figure 2. The position of blade 1 and the PIV sheet for azimuthal position of $\Psi=0^{\circ}$. Main flow axis is into the sheet.

\subsection{AD model implemented in FLUENT}

The actuator disk formulation was coupled to FLUENT by so-called USER DEFINED FUNCTIONS. To distinguish between the outer and AD flow, a special domain had to be defined where the momentum sources was introduced. The mesh had about one million hexahedral cells [3].

The Actuator Disk separates sources in $\mathrm{x}-$, $\mathrm{y}$ - and $\mathrm{z}$-direction. For each source the radial position was defined and an aerodynamic state (induction, velocities and angle of attack) was computed. With this data and the radius the lift and drag coefficients $\left(c_{l}, c_{d}\right)$ were determined for the blade section in the current cell. The coefficients were computed from polynomial approximations that described the aerodynamic behavior. Lift and drag were corrected and transferred from cylindrical to Fluents Cartesian coordinates. The sum of the forces are corresponding to thrust and torque.

In the hub region $r<r_{H u b}$ the lift coefficient was set to zero and the drag coefficient was assumed to be in the order of $\left(c_{d}=1.3\right)$.

The details of this actuator disk model as well as the results were explained in detail in $[3,5]$. The AD model for OpenFOAM was based on this model and is described in section 3. The computations were performed without any turbulence modeling, because - in priciple - AD models are assumed to be inviscid. 


\subsection{Blade element method (BEM)}

A more advanced analysis of the aerodynamic behavior of horizontal axis wind turbine include the blade element method (BEM) in addition to the analysis of energy extraction process examined by the actuator disk model. The blade element method allows to consider the effects of rotor geometry characteristics like chord and twist distributions of the blade airfoil. This method is based on the assumptions that there is no aerodynamic interactions between different blade elements and the force on the blade elements are solely determined by the lift and drag coefficients. The blade is separated into radial sections [6] since each of the blade elements has a different rotational speed and geometric characteristics hence experiencing a slightly different flow. This method is simplified by defining radial sections by well known aerodynamic profiles (like NACA 64-418, Risoe A1-21 and Du91-W2-250 profiles). Lift and drag coefficients are then extracted from a look-up table for each airfoil section for the current angle of attack. With these informations the local forces on the blade can be computed for a specified inflow velocity, tip speed ratio and pitch angle. The flow is assumed a slip stream that is well defined by momentum theory [6] since the flow field around the rotor is not available. Therefore, this model will not be considered in the comparison of velocities in this study. The global forces thrust and torque are then extrapolated from the local forces where the overall performance characteristics of the blade can be determined. The principal points are summarized hereafter.

\subsection{FUII 3D RANS modeling using DLR's tau-Code}

Next to the model approaches described before, a direct modeling with computational fluid dynamics (CFD) was also done for the MEXICO rotor. Therefore, the rotor was separated into $120^{\circ}$ sections containing only a single blade (Fig. 1). The hub and nacelle were also included in the geometry.

An unstructured mesh was used for the flow modeling. It was built up with 1.26 million nodes (6.12 million cells). The blade was resolved by 141,000 triangle elements on the surface and with three layers of prism cells to resolve the wall boundary. The Spalart-Allmaras turbulence model [7] was used to estimate the influence of turbulence.

The DLR Tau Code [8] was used for the computations. It is a compressible RANS solver that is commonly applied in the aerospace industry. The code supports a wide range of Mach numbers and provides a preconditioning for low Mach numbers. This technique is used in these simulations.

To include the rotational effects of the wind turbine, DLR Tau Code provides movement for meshes. A rotation around the X-axis was set up with $424 \mathrm{RPM}$. The rotational effects by the two other sections that were not modeled were represented by rotational periodic boundaries (blue planes in Fig. 3).

The results of this and other CFD simulations as well as other modeling approaches were compared to the measurements in the MexNext project. A detailed discussion can be found in [9].

\section{Tabulated actuator disk model in OpenFOAM}

For the implementation of the actuator disk model in OpenFOAM the work of Svenning [2] provided the frame. The SIMPLE solver routine simpleFoam was used, too. To the momentum equation the source term $\vec{S}_{A D}$ was added. This source term was determined by the forces which act on the fluid at the actuator disk. The momentum equation was:

$$
\frac{\partial \vec{U}}{\partial t}+\nabla \cdot(\vec{U} \vec{U})-\nabla \cdot(v \nabla \vec{U})=-\frac{1}{\rho} \nabla p+\vec{S}_{A D}
$$

where $\vec{U}$ was the velocity, $t$ was the time, $p$ the pressure. The density $\rho$ was constant throughout the computational domain. 


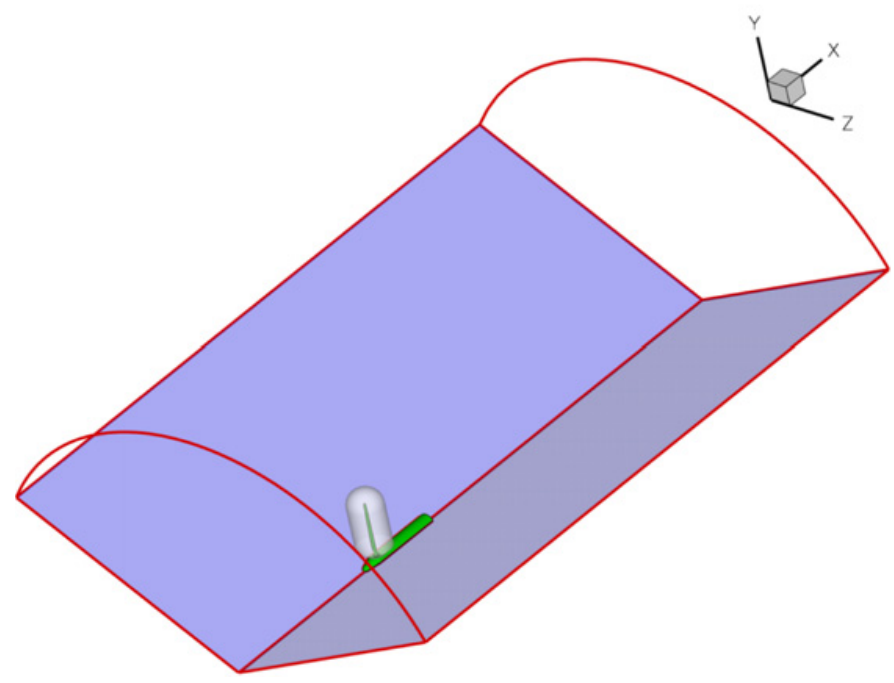

Figure 3. $120^{\circ}$ section used to model the MEXICO rotor in DLR Tau Code. Blue planes are rotational periodic boundaries of the computational domain. The transparent bulb shows the region of high mesh density to sufficiently resolve the flow near the blade.

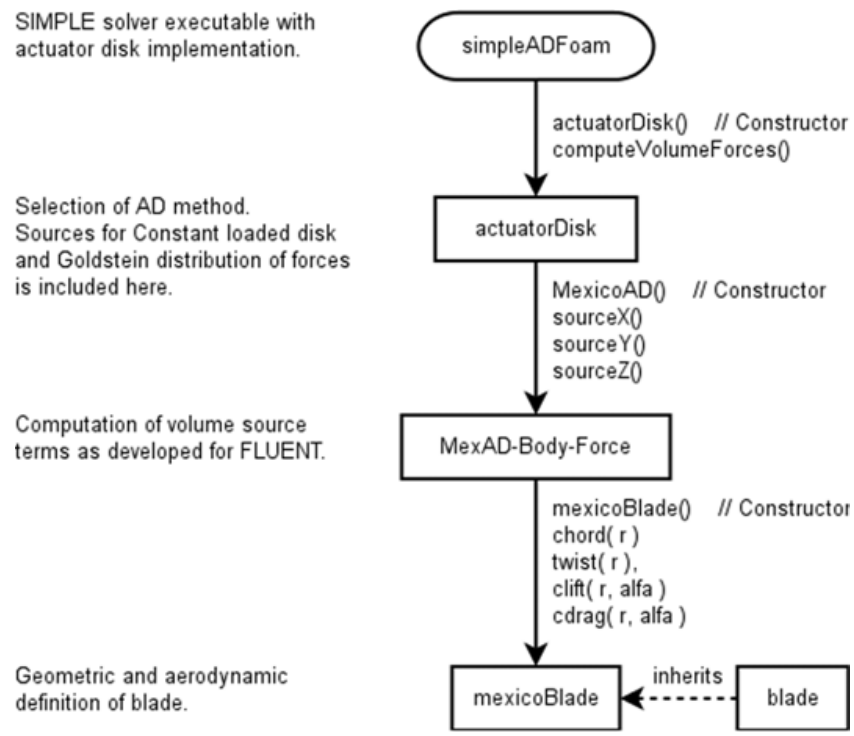

Figure 4. List of Classes and Files used in the implementation. Arrows show dependencies on classes. The used methods are shown next to the dependency arrows.

In the original program was the volume force $\vec{S}_{A D}$ included in the arguments of the solve-function, but it had to be included in the definition of the vector-matrix UEqn [10]. Thus, the volume forces would be considered in the preconditioning.

The volume forces were computed in the class actuatorDisk (see Fig. 4). This class identified the mesh cells that were inside the AD volume and added the volume forces $\vec{S}_{A D}$. Here, a validation model with a constant loaded rotor was easy to include, but a real wind turbine like the MEXICO rotor would need a more sophisticated model. Therefore, the parametric AD model for Fluent [3] was added as the 


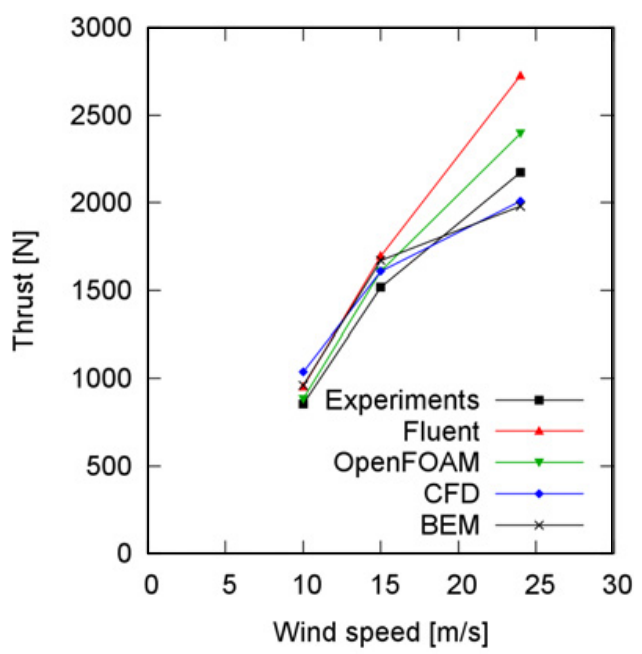

Figure 5. Thrust for all three test cases.

class MexAD-Body-Force. This class provided the methods with the source terms in the three directions $x, y$ and $z$. These functions computed local radius $r$ and angle of the current cell as well as the velocity in cylindrical coordinates. The velocities were corrected near tip and hub and the angle of attack $\alpha$ was determined.

With $r$ and $\alpha$ were the geometric and aerodynamic properties of the airfoil determined. These properties were represented as parametric functions in the original model. However, the determination of these functions was time consuming and error-prone. Therefore, it was decided that the AD model for OpenFOAM should read the geometric and aerodynamic data directly from files, where this data was stored in tabulated format. Linear interpolation was used between two data points. This part was provided by the class blade.

But the MEXICO rotor was special, because it was made from three different airfoils (Fig. 1). Therefore, the class mexicoBlade was created. It provided the same public methods as the class blade, but using the radius $r$ in the function call, the lift and drag coefficients could be computed for the correct airfoil. Linear interpolation was used again, when $r$ was in the transfer zones between two defined airfoils.

Using tabulated airfoil data and determining the volume forces from aerodynamic properties instead of prescribed thrust and torque opened a broad field of applications for this model, including the simulation of wind farms and wind shear.

\section{Results}

The reference cases and our new actuator disk model in OpenFOAM are compared now. The lines are black with squares for the experiments, read with triangle up for Fluent with parametric actuator disk model, green with triangle down for OpenFOAM and the tabulated actuator disk model, blue with diamond for direct model using CFD and black with crosses for BEM model. From all available data a small selection was chosen to represent the outcome.

\subsection{Global (integrated) values for Thrust \& Torque}

The overall information on thrust and torque was available for all models. In Figure 5 the thrust is shown over the wind speed for the three test cases. At wind speeds of of $10 \mathrm{~m} / \mathrm{s}$ and $15 \mathrm{~m} / \mathrm{s}$ the results for all 


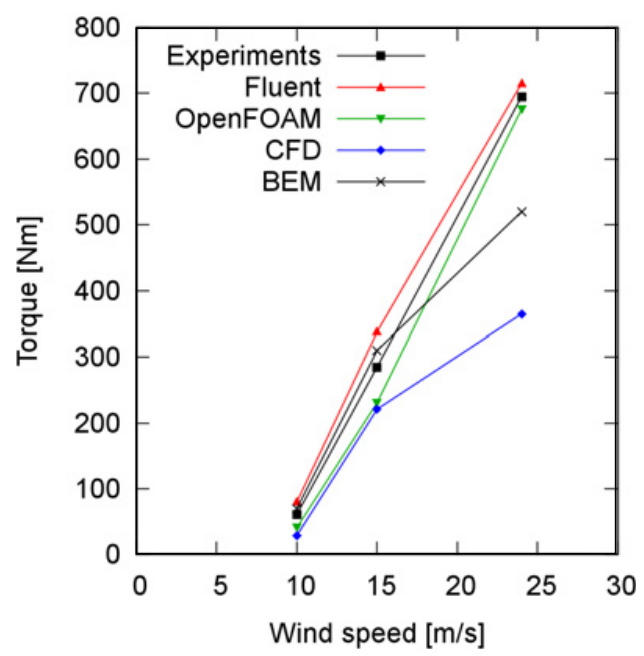

Figure 6. Torque for all three test cases.

models were close together and in agreement with the experiments. At $24 \mathrm{~m} / \mathrm{s}$ wind speed case, wide spreading of the predicted thrust occurred. The direct modeling and BEM under-predicted the thrust while both AD models computed values that were to large. The actuator disk model in OpenFOAM performs better than the parametric model in Fluent.

In Figure 6 the results for the torque are shown. For the low wind speed of $10 \mathrm{~m} / \mathrm{s}$ the torque from experiments and models were in agreement. But at $15 \mathrm{~m} / \mathrm{s}$ the models deviate from the experiments. The tabulated AD model and direct modeling under-predicted the torque and BEM and parametric model over-predicted the torque. The deviations became worse for BEM and direct model at $24 \mathrm{~m} / \mathrm{s}$. But both $\mathrm{AD}$ models were very close to the experimental value with their predicted torque.

\subsection{Blade loads}

While integrated thrust and torque values could be provided by all models, the blade performance could be seen with the local loads on the blade. The normal force in Figure 7 was normal to the local chord axis of the airfoil, the tangential force was along the local chord axis.

At $10 \mathrm{~m} / \mathrm{s}$ case (Fig. 7a) and at design conditions $(15 \mathrm{~m} / \mathrm{s}$ case, Fig. 7c) the normal forces of all models were in agreement with the experimental values. But all models predicted too high values for the forces. Only the tabulated AD model in OpenFOAM did not represent the curves exactly. The slope was not as steep as experiments and other models predicted. At design conditions the predicted forces were too high at the inner part of the blade.

At $24 \mathrm{~m} / \mathrm{s}$ case (Fig. 7e) the results were much different. The parametric AD model in Fluent was different than the experimental values. The new tabulated AD model in OpenFOAM was representing the experimental curve quite well, also the total values were predicted too high.

The tangential forces in direction of the local chord axis are displayed in Figures $7 \mathrm{~b}, \mathrm{~d}$ and $\mathrm{f}$. At $10 \mathrm{~m} / \mathrm{s}$ and $15 \mathrm{~m} / \mathrm{s}$ case the direct model was able to reproduce the tangential forces alone. The tabulated AD model in OpenFOAM was not in agreement with the experimental curves, so further improvement is necessary. 


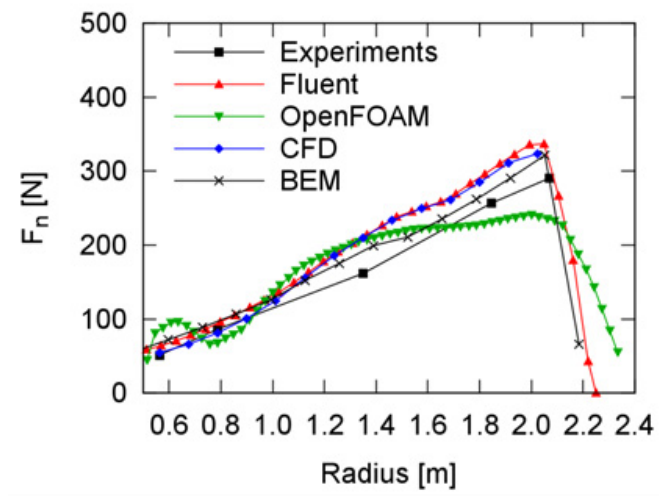

(a) Normal force, $10 \mathrm{~m} / \mathrm{s}$

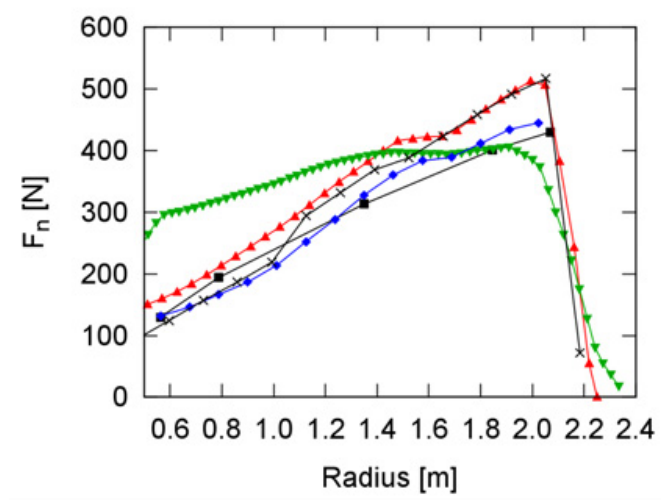

(c) Normal force, $15 \mathrm{~m} / \mathrm{s}$

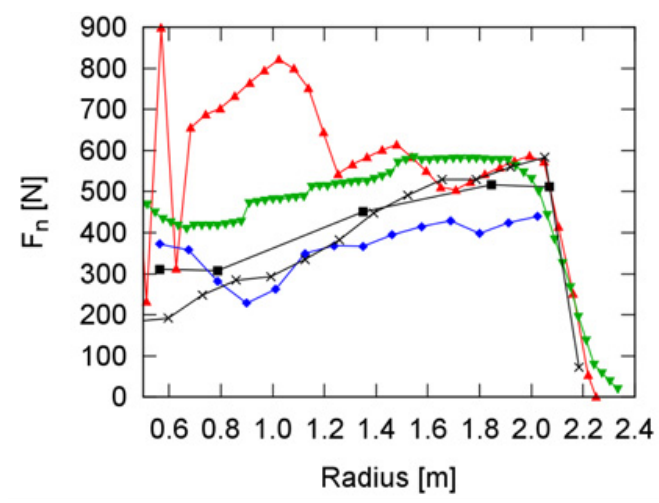

(e) Normal force, $24 \mathrm{~m} / \mathrm{s}$

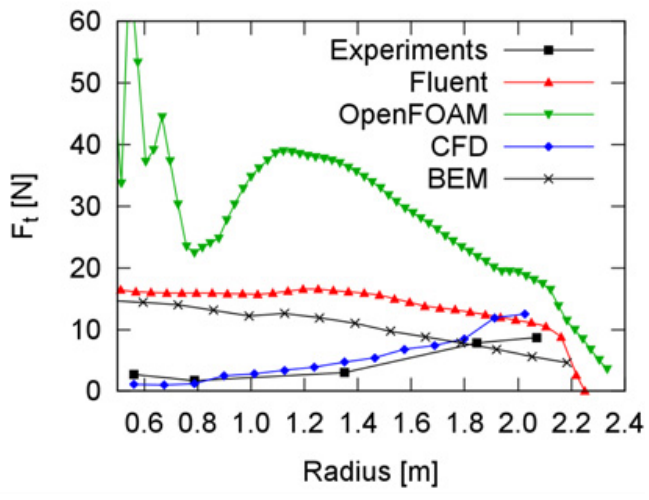

(b) Tangential force, $10 \mathrm{~m} / \mathrm{s}$

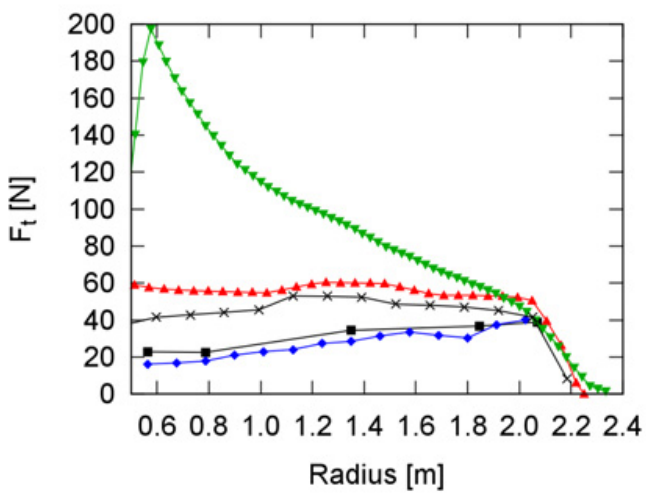

(d) Tangential force, $15 \mathrm{~m} / \mathrm{s}$

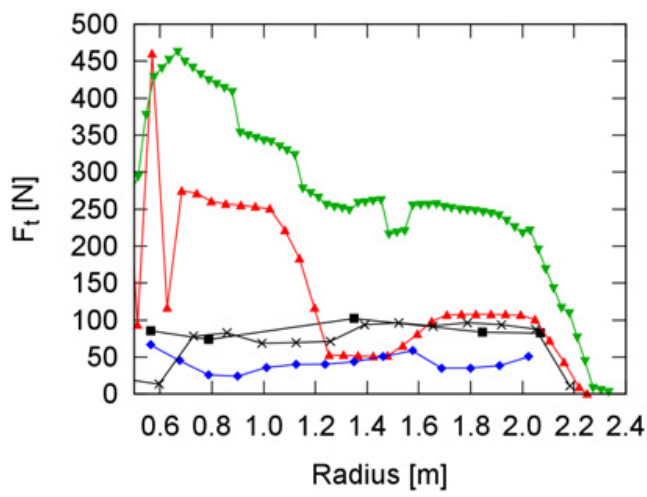

(f) Tangential force, $24 \mathrm{~m} / \mathrm{s}$

Figure 7. Normal (left) and tangential (right) loads on the blade over the radius.

\subsection{Near-by FLOW field: Axial velocity profiles}

One application of actuator disk models was the design of wind farms, so the correct representation of the velocity profiles would be more important than the overall and local forces. The BEM model 
First Symposium on OpenFOAM in Wind Energy

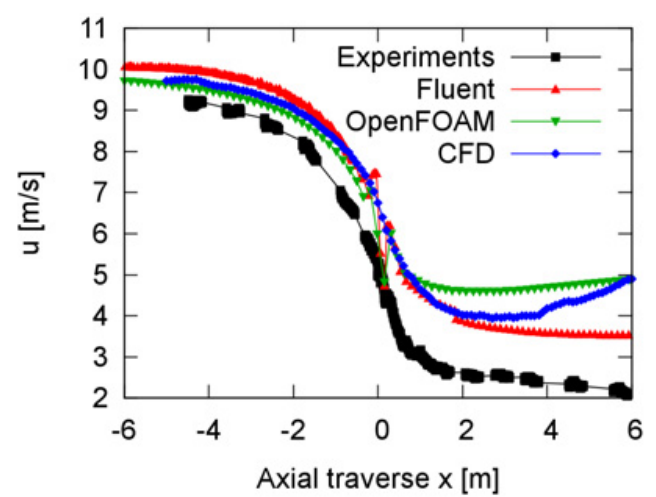

(a) u@ $10 \mathrm{~m} / \mathrm{s}$

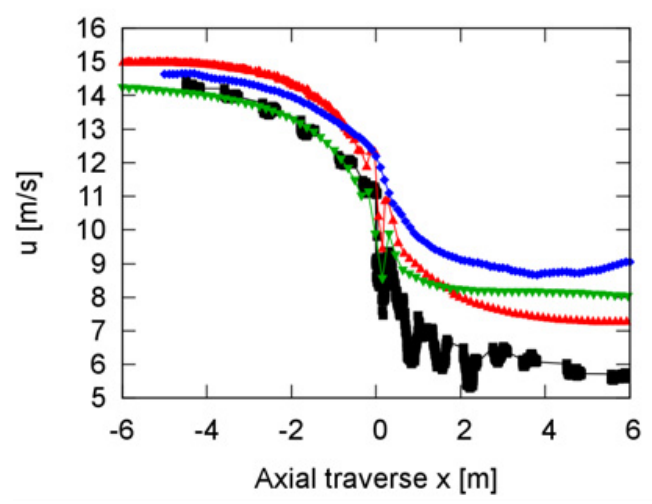

(c) u@ $15 \mathrm{~m} / \mathrm{s}$

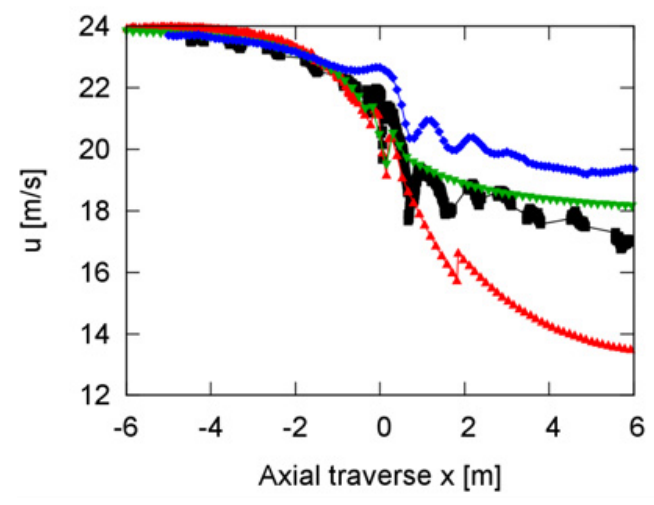

(e) u@ $24 \mathrm{~m} / \mathrm{s}$

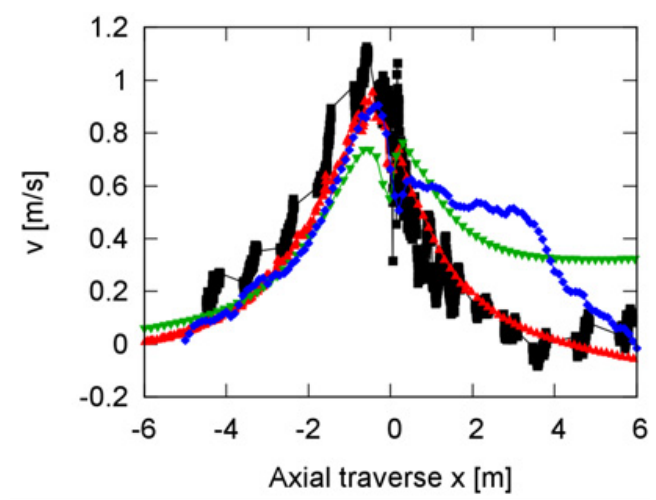

(b) v@10 m/s

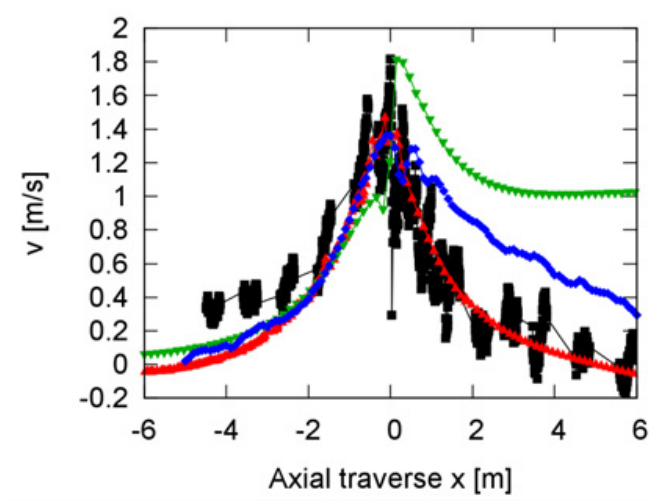

(d) $\mathrm{v} @ 15 \mathrm{~m} / \mathrm{s}$

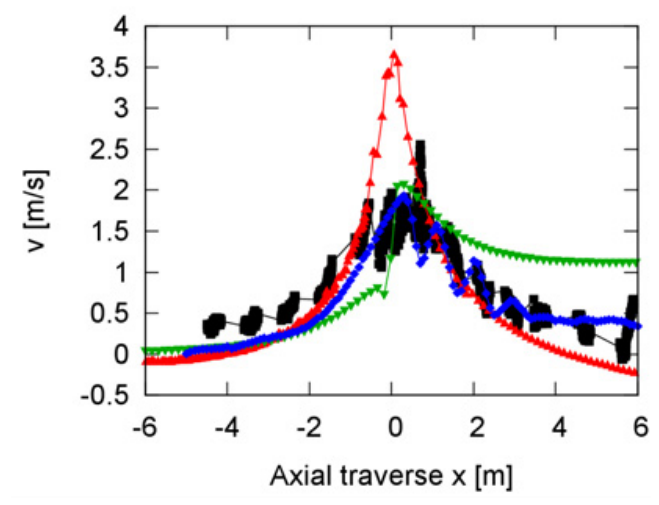

(f) $\mathrm{v} @ 24 \mathrm{~m} / \mathrm{s}$

Figure 8. Axial velocity profiles at $\mathrm{r}=1.37 \mathrm{~m}$ and $\Psi=0^{\circ}$.

assumed a stream tube for the wind speed and used the approximated velocities for the computation of loads and performance, so no real information on the surrounding field was available.

The axial traverses for each case are shown in Figure 8 with the axial velocity $u$ on the left and the radial velocity $v$ on the right. The rotor was located at $x=0 \mathrm{~m}$. In comparison to the experiments all 
three models had an offset for the inflow velocity in $10 \mathrm{~m} / \mathrm{s}$ and $15 \mathrm{~m} / \mathrm{s}$ cases at $x=4 \mathrm{~m}$. However, the inflow velocities were the same value for all models.

For the $10 \mathrm{~m} / \mathrm{s}$ case (Fig. 8a) the axial velocity behind the rotor was predicted too high by all models. Direct model and parametric model reached an induction factor $a=v_{\text {out }} / v_{\text {in }}=1 / 3$. The rise of the velocity in the direct model beginning at $x=2 \mathrm{~m}$ was due to a rather coarse mesh in the wake region. For the experimental velocities an induction factor $a=0.27$ was found, which is not in agreement with the stream tube theorem. Additional blockage effects of the wind tunnel might have caused this [9].

For the design conditions at $15 \mathrm{~m} / \mathrm{s}$ (Fig. 8c) the induction factors were about $a=0.4$ for the experiments, for the models $a$ was between 0.48 and 0.56. In Figure 8e the offset was less important, the results downstream of the rotor were wide spreading. The tabulated model had the best result.

The prediction of the radial velocity component $v$ (Figs. $8 \mathrm{~b}$ and $\mathrm{d}$ ) was best represented by the parametric model. Both direct model and tabulated AD model were over-predicting the rotational effects downstream of the rotor. In Figure $8 \mathrm{f}$ the direct model was matching the experimental values accurately near the rotor and downstream.

\subsection{Radial velocity profiles}

The radial velocity profiles of the axial velocity u for an azimuth angle $\Psi=0^{\circ}$ (see Fig. 2) are shown in Figure 9. The upstream velocity profiles for each case are on the left side and the downstream profiles are on the right side.

The offset in inflow velocity that was found for the axial traverses, could be seen here, too. Especially for the $10 \mathrm{~m} / \mathrm{s}$ case (Figs. 9a and b). The qualitative prediction of the flow field was in agreement with the experimental data.

For $15 \mathrm{~m} / \mathrm{s}$ case (Figs. 9c and d) both actuator disk models performed well. The parametric model predicted the small difference in velocity at $r=1.6 \mathrm{~m}$ better, the tabulated model performed better near the blade tip.

The smooth run of the curve at $24 \mathrm{~m} / \mathrm{s}$ inflow case (Figs. 9e and f) was best represented by the direct model, but the velocities were too high. The actuator disk models had again the steep rise of the velocity near the blade tip at $r=2.25 \mathrm{~m}$. The drop in velocity at the inner blade region for the parametric AD model was probably caused by an error in one of the parametric functions.

\subsection{Comments on possible reasons for the differences in numerical results}

As may be seen from the the preceeding sub-sections the differences are rather large and generally increasing with wind-speed. First of all it seems hard to say which of the values (even the measurement) should serve as a reference for quantifying the inaccuracies. The $25 \mathrm{~m} / \mathrm{s}$ case has to be discussed separately, because in this case most of the flow is separated and the differces may indicate the quality of modelling this type of - highly transient - flow. Coming back to the 10 and $15 \mathrm{~m} / \mathrm{s}$ cases the radial resolved loads (Fig. 7) show reasonalbe agreement except for the data from OpenFOAM (green dots). The peak at low an medium radial stations of $F_{t}$ in Figures $7 \mathrm{~b}$ and d indicates probably wrong data processing, because the torque is comparable to the CFD (=tau - code) data.

Regarding the velocity data (Fig. 8) it is remarkable that the u (axial, windward) components from numerical simulation after the disk seem to be much lower that measured. Again the $24 \mathrm{~m} / \mathrm{s}$ case shoud be regarded differently. The slight increase of $u$ in Figures 7a, $c$ and e indicates incomplete convergence of the RANS-CFD (tau-code)solution.

It has to be noted that the differences shown here agree well with those from other numerical investigations as reported in [9]. 
First Symposium on OpenFOAM in Wind Energy

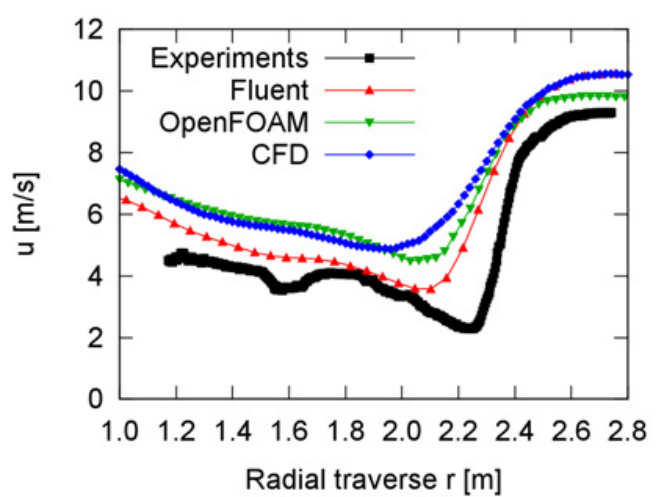

(a) u@ $10 \mathrm{~m} / \mathrm{s}$, upstream

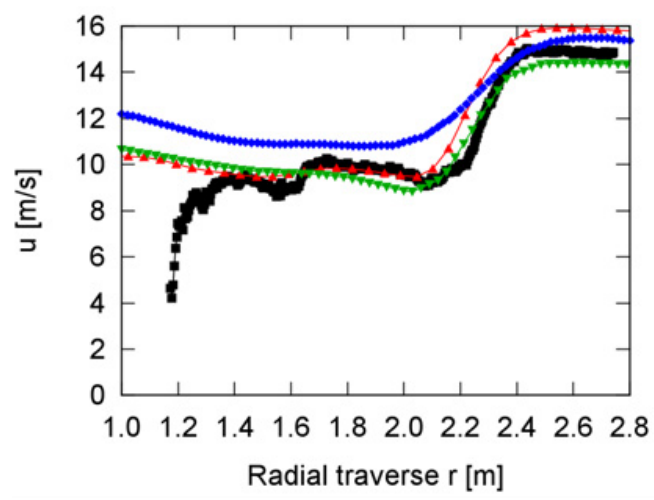

(c) u@15 m/s, upstream

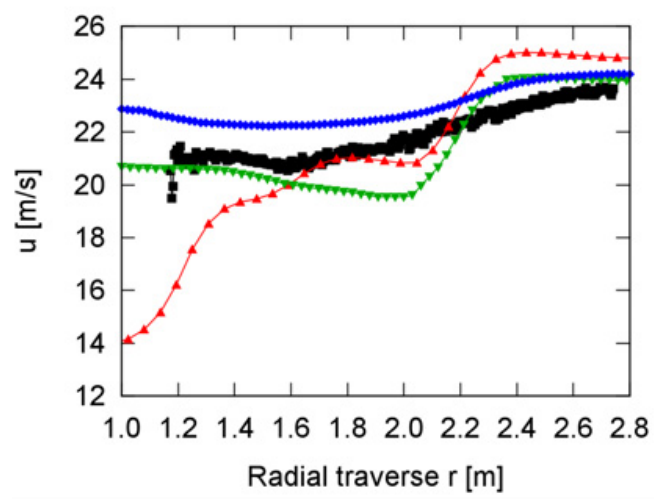

(e) u @ $24 \mathrm{~m} / \mathrm{s}$, upstream

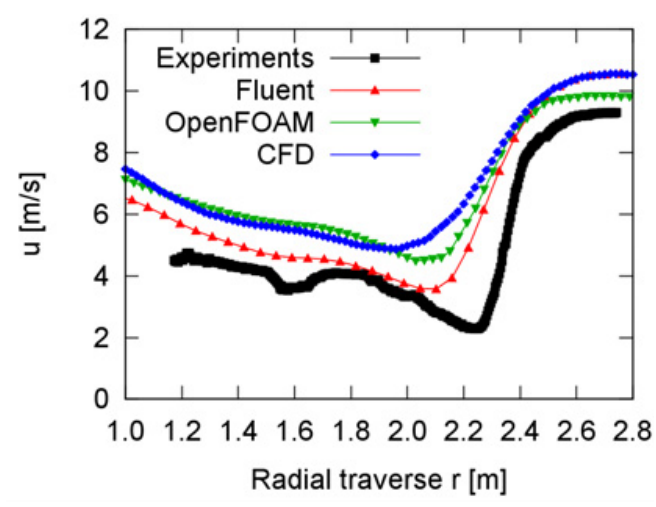

(b) u @ $10 \mathrm{~m} / \mathrm{s}$, downstream

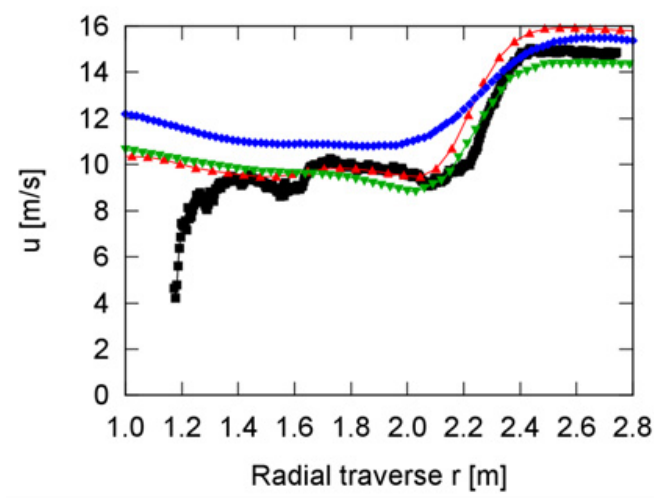

(d) u@ $15 \mathrm{~m} / \mathrm{s}$, downstream

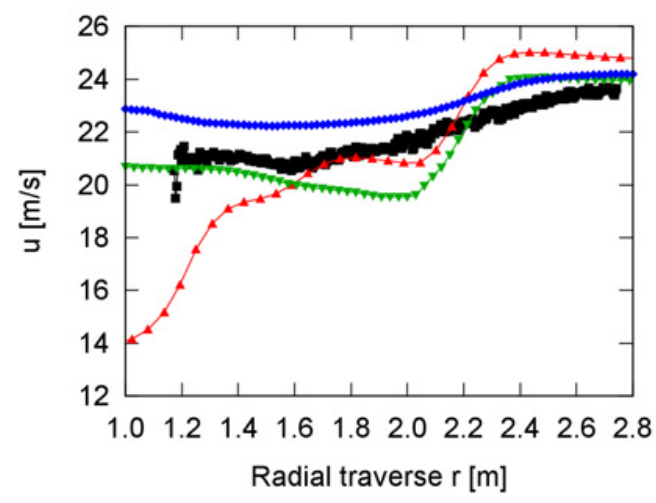

(f) u @ $24 \mathrm{~m} / \mathrm{s}$, downstream

Figure 9. Radial velocity profiles upstream $(x=-0.3 \mathrm{~m})$ and downstream $(x=0.3 \mathrm{~m})$ at azimuth angle $\Psi=0^{\circ}$.

\section{Conclusions}

The actuator disk model of Svewnning was improved for use of the MEXICO Rotor in OpenFOAM version 2.1.1. 
A detailled comparison was given to a simple BEM-Model, an AD formulation using a commerical code (FLUENT) a full CFD-RANS model (tau-code) and experimental data. This comparison was particular interesting, because velocity measurements were available for the first time in wind turbine rotor aerodynamics.

The prediction of the overall thrust and torque was in the same accuracy band as the other methods and close to the data from experiments. Regarding the spanwise distribution of normal and tangential forces marked differences are visible. The prediction of velocity-distributions were in reasonalbe agreement to the experiments as well.

As was previously noted by other investigators, BEM and AD methods use tabulated 2D lift- and drag-data from airfoils which may not remain valid in the separated case $(25 \mathrm{~m} / \mathrm{s}$ inflow velocity). In addition to that the degree of accuracy of the experimental data was discussed extensively during the IEAwind's MexNext task (phase 1). Regarding further development of AD-methods for use in openFOAM is has to be noted, that the method used for solving the flow field could be simplifyed to potential flow in principle reducing the CPU-time for solving significantily.

\section{References}

[1] A. Schaffarczyk, Introduction to Wind Turbine Aerodynamics (Springer Verlag, Berlin, Heidelberg, Germany, 2014)

[2] E. Svenning, Implementation of an actuator disk in OpenFOAM, Bachelor thesis (Chalmers University of Technology, Sweden, 2010)

[3] E. Mahmoodi, A. Schaffarczyk, J. Mahmoudi, Actuator Disk Modeling of the Mexico Rotor, in EUROMECH Colloquium 528: Wind Energy and the impact of turbulence on the conversion process, Oldenburg, Germany (2012)

[4] J. Schepers, H. Snel, Tech. rep. (2007), ECN-E-07-042

[5] E. Mahmoodi, A. Schaffarczyk, J. Mahmoudi, Incompressible Navier-Stokes against RANS Turbulence Model for wake Modelling via Actuator Disc Theory using FLUENT, in Proc. The science of making torque from wind, Oldenburg, Germany (2012)

[6] R.E. Wilson, P.B.S. Lissaman, S.N. Walker, Tech. rep., Oregon State Univ., Corvallis (USA) (1976)

[7] P.R. Spalart, S.R. Allmaras, A one-equation turbulence model for aerodynamic flows, AIAA-920439 (1992), 30th Aerospace Sciences Meeting \& Exhibit, Reno, NV

[8] D. Schwammborn, T. Gerhold, R. Heinrich, The DLR TAU-Code: Recent Applications in Research and Industry, in European Conference on Computational Fluid Dynamics ECCOMAS CFD (2006)

[9] J. Schepers, K. Boorsma, T. Cho, S. Gomez-Iradi, P. Schaffarczyk, A. Jeromin, W. Shen, T. Lutz, K. Meister, B. Stoevesandt et al., Tech. rep. (Feb. 2012), ECN-E-12-004

[10] J. Nathan, Private Communication (2012) 